\title{
Combined Presigmoid and Retrosigmoid Approach to Petroclival Meningiomas
}

\author{
Marcio S. Rassi ${ }^{1}$ Johnni O. Zamponi jr ${ }^{1} \quad$ Duarte N. C. Cândido ${ }^{1} \quad$ Jean G. de Oliveira ${ }^{2,3}$ \\ Gustavo A. R. Passos ${ }^{1}$ Luis A. B. Borba ${ }^{1,4}$ \\ ${ }^{1}$ Department of Neurosurgery, Evangelic University Hospital of \\ Curitiba, Parana, Brazil \\ ${ }^{2}$ Division of Neurosurgery, Department of Surgery, Santa Casa de São \\ Paulo School of Medical Sciences (FMSCSP), São Paulo, Brazil \\ ${ }^{3}$ Division of Cerebrovascular and Skull Base Surgery, Center of \\ Neurology and Neurosurgery Associates (CENNA), Hospital \\ Beneficência Portuguesa de São Paulo, São Paulo, Brazil \\ ${ }^{4}$ Department of Neurosurgery, Federal University of Paraná, Curitiba, \\ Parana, Brazil \\ J Neurol Surg B 2018;79(suppl S5):S402-S403.

\begin{abstract}
Address for correspondence Luis A. B. Borba, MD, PhD, IFAANS, Rua Paulo Gorski 1240 7, CEP 81210220, Curitiba, PR-Brazil

(e-mail: luisborba@me.com).
\end{abstract}

\begin{abstract}
Introduction The management of petroclival meningiomas is among the most intimidating in neurosurgery, due to its difficult accessibility and close relationship with vital structures; therefore, an appropriate exposure is mandatory. We present a surgical video demonstrating a presigmoid transtentorial approach, associated with the opening of the retrosigmoid dura to a petroclival meningioma, performed by the senior surgeon (L.A.B.B.), along with its indications and pitfalls.

Approach Decision The patient's clinical history is summed to the tumor's radiological features as its extension, vascularization, and venous drainage, when selecting the appropriate approach. The presigmoid transtentorial approach offers a wide exposure of the petroclival area along with the tumor's attachment. Its association with the retrosigmoid route allows the surgeon to freely work through multiple paths, and

Keywords

- meningioma

- petroclival

- petrosal

- skull base

- brain tumor parallel to the skull base, reducing the traction in the temporal lobe.

Clinical Case This is a 39-year-old female presenting with trigeminal neuralgia. Imaging depicted a petroclival meningioma, extending from the posterior aspect of the cavernous sinus to the cerebellopontine angle, extending inferiorly to the jugular bulb. A Simpson II resection was achieved through a combined presigmoid and retrosigmoid approach, and the patient was discharged with no complications or new deficits.
\end{abstract}

Conflict of Interest

None.

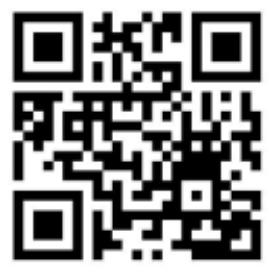

received

May 29, 2018

accepted

August 13, 2018

published online

September 25, 2018 www.thieme.com/skullbasevideos

www.thieme.com/jnlsbvideos

DOI https://doi.org/

10.1055/s-0038-1669965.

ISSN 2193-6331. (c) 2018 Georg Thieme Verlag KG

Stuttgart · New York
License terms

(c) (1) $\ominus$ (\$) 
Conclusion Petroclival meningiomas are a formidable and surgically treatable disease. The appropriate approach is design to each patient and should not be the limiting factor for total tumor removal, which is truly given by the absence of a dissection plane between the tumor and the brainstem, nerves and vascular structures of the skull base. The link to the video can be found at: https://youtu.be/MFjqZvElBSo. 